\title{
CONSTRUYENDO LA PAZ. ANÁLISIS COMPARATIVO DE LA CONTENGIÓN DEL CRIMEN ORGANIZADO EN CHICAGO, SICILIA Y LA LAGUNA
}

\author{
CONSTRUCTING PEACE: COMPARATIVE ANALYSIS \\ OF THE CONTAINMENT OF ORGANIZED CRIME IN \\ CHICAGO, SICILY AND LA LAGUNA
}

CONSTRUIRE LA PAIX. ANALYSE COMPARATIVE DE LA RÉDUCTION DE LA CRIMINALITÉ ORGANISÉE À CHICAGO, EN SICILE ET À LA LAGUNA

\author{
Sergio Aguayo Quezada \\ El Colegio de México \\ saguayo@colmex.mx \\ Rodrigo PeÑa GonzÁlez \\ El Colegio de México \\ rpena@colmex.mx
}

RESUMEN: ¿Existe algún modelo o fórmula de validez universal para contener al crimen organizado? Este artículo aborda esta pregunta clave comparando tres experiencias de contención: Chicago, EE.UU. (en la primera mitad del siglo xx), Sicilia, Italia (en las últimas décadas del mismo siglo xx y primeras del Xxi), y La Laguna, Coahuila y Durango (desde 2007). En ellos se detectan y destacan patrones de reacciones y coordinaciones sociales ante problemáticas similares de violencia y gobernanza del crimen. El artículo comienza estableciendo una discusión conceptual sobre la gobernanza criminal y el capital social. Posteriormente compara con las mismas variables los tres casos y finaliza señalando las similitudes, para cerrar con la discusión en torno a la pregunta de investigación planteada al inicio.

Palabras clave: capital social; Chicago; Sicilia; La Laguna; crimen organizado; gobernanza.

ABstRACT: Is there a valid universal model or formula to contain organized crime? This paper addresses this key question by comparing three experiences 
of containment: Chicago (in the first half of the $20^{\text {th }}$ century), Sicily (in the late $20^{\text {th }}$ century and early $21^{\text {st }}$ century) and La Laguna (since 2007). Patterns of social reaction and coordination in response to similar problems of violence and governance of crime are identified and highlighted. The paper begins with a conceptual discussion of criminal governance and social capital before using these variables to compare the three cases. It moves on to identifying the similarities between them before closing with a discussion of the research question proposed at the outset.

Keywords: social capital; Chicago; Sicily; La Laguna; organized crime; governance.

\section{Traducción de Fionn Petch, CM Idiomas}

RÉsumÉ: Existe-t-il un modèle ou une formule universellement valable pour contenir le crime organisé? Cet article aborde cette question clé en comparant trois expériences d'endiguement: Chicago (dans la première moitié du $20^{\mathrm{e}}$ siècle), la Sicile (dans les dernières décennies de ce même $20^{\mathrm{e}}$ siècle et les premières décennies du 21e) et La Laguna, au Mexique (depuis 2007). Dans celles-ci, des modèles de réactions et de coordination sociale face à des problèmes similaires de violence et de gouvernance de la criminalité sont décelés et mis en relief. L'article commence par établir une discussion conceptuelle sur la gouvernance criminelle et le capital social. Par la suite, il compare les trois cas avec les mêmes variables et termine en soulignant les ressemblances avant de conclure par une discussion autour de la question de recherche posée au départ.

Mots-clés: capital social; Chicago; Sicile; La Laguna; crime organisé; gouvernance.

Traducción de Rafael Segovia, CM Idiomas

Fecha de recepción: febrero de 2021

Fecha de aceptación: mayo de 2021 


\section{INTRODUCGIÓN}

Existe algún modelo de validez universal para contener la violencia que produce el fenómeno del crimen organizado? La repuesta es sí, existe, y para acercarse a él se debe trascender el mito de "los intocables", reconfirmado por Hollywood en la película del mismo nombre (Brian De Palma, 1987), un espectáculo cautivador que durante 119 minutos tuerce y retuerce el cuello del rigor histórico. Quienes la hayan visto recordarán que el relato gira en torno al conflicto entre Al Capone y Eliot Ness; el gangster y el policía. Nunca aparece la dama o la diva simbolizando a "doña Sociedad Civil”, una figura clave en el Chicago de la prohibición.

Esta omisión fue recordada por el poeta Javier Sicilia una tarde de mayo de 2011. Ante un Zócalo repleto, en la Ciudad de México, lanzó un reto: "Estamos aquí para decirnos y decirles que este dolor del alma en los cuerpos [...] lo convertiremos [...] en una palanca que nos ayude a restaurar el amor, la paz, la justicia, la dignidad y la balbuciente democracia que estamos perdiendo". ${ }^{1} \mathrm{Y}$ el movimiento que encabezó metió el tema de las víctimas en la agenda nacional, tal y cómo lo habían hecho otros afectados por la violencia en Sicilia, Italia, en décadas anteriores.

Excluir a la sociedad es un actitud muy frecuente pese a la importancia de la participación de seis actores sociales para contener los estragos de grupos criminales violentos: empresarios, medios de comunicación independientes, colectivos de víctimas, organismos de la sociedad civil, grupos religiosos y académicos. ${ }^{2} \mathrm{Su}$ influencia puede transformarse en política pública cuando se enlaza con los funcionarios públicos comprometidos con el combate a los delincuentes.

${ }^{1}$ Elena Azaola, "La violencia de hoy, las violencias de siempre”, Desacatos, 40, septiembre-diciembre (2012), pp. 159-170.

${ }^{2}$ Como se verá en la revisión de los casos, estos seis actores eventualmente se complementan con la participación de funcionarios públicos comprometidos con el combate del grupo criminal local. 
En los casos aquí revisados, cada uno de estos seis actores respondía a impulsos, intereses y relatos diversos, conectados por el deseo de enfrentar al crimen organizado que había establecido una gobernanza criminal en las tres localidades mencionadas. Para enfrentarla, se acercaron a las autoridades federales, estatales y locales, y acordaron estrategias exitosas.

Este texto da inicio con una discusión teórica sobre la gobernanza criminal y el capital social. Posteriormente, ordenamos la información sobre los tres casos de estudio en tres etapas: la coexistencia con el crimen, el punto de quiebre asociado a un hecho particularmente violento y la aplicación de estrategias públicas consensuadas entre Estado y sociedad civil con final exitoso. Por último, cerramos el artículo con una pregunta clave que, quienes firmamos este texto, buscamos responder con investigaciones conducidas desde el Seminario sobre Violencia y Paz de El Colegio de México: ¿puede reproducirse este modelo en la Ciudad de México y en otros centros urbanos?

\section{LA GOBERNANZA GRIMINAL Y EL CAPITAL SOGIAL}

Esta sección debería empezar con la discusión del "capital social”, porque las personas primero se organizan y después deciden si apoyan al crimen organizado, al Estado de derecho o se convierten en observadores pasivos. Empezamos con gobernanza criminal porque, en los tres casos, la sociedad organizada reaccionó ante la presencia criminal cuando ésta rebasó los límites de violencia tolerables que, como veremos más adelante, varían de acuerdo con el país y la región geográfica.

Definir el crimen organizado es un asunto problemático. ${ }^{3}$ Detengámonos en la definición del Federal Bureau of Investigation (FBI):

${ }^{3}$ Klaus von Lampe (comp.), Definitions of Organized Crime (sitio de internet), www.organized-crime.de/organizedcrimedefinitions.htm 
Cualquier grupo de seis o más personas, en que alguno de los seis ocupa un rol de coordinador, cuyo principal objetivo sea obtener dinero mediante actividades ilegales. Estos grupos usan las siguientes actividades para mantener su poder: violencia física, o amenazas de llevarla a cabo, corrupción de funcionarios públicos, infiltración o extorsión, y por lo general, sus actividades tienen un impacto en las poblaciones, ya sea en el ámbito local, regional o en todo el país. ${ }^{4}$

Dejan la sensación de que lo conciben como un actor diferenciado del resto de la sociedad y de las autoridades oficiales. No es el caso. Las mafias y los carteles son parte de sus respectivas sociedades y cuentan con la protección de una parte del Estado. Por ello, consideramos más preciso utilizar el término de gobernanza criminal: da protagonismo a estos grupos porque establecen redes con actores legales e ilegales y crean una gobernanza desde la ilegalidad sobre una parte de la población.

De acuerdo con Enrique Desmond Arias, es común que los grupos criminales en Latinoamérica y el Caribe "operen en el contexto del funcionamiento de instituciones estatales". ${ }^{5}$ En otras palabras, las autoridades legales y oficiales coexisten con estos grupos criminales. Esta convivencia dependerá, en lo local, del tipo de gobernanza criminal, del grado de consolidación criminal y del uso de la violencia.

Para Benjamin Lessing, la gobernanza criminal es "la imposición de reglas o la restricción del comportamiento por parte de una organización criminal" en un territorio específico. ${ }^{6}$ Ello implica la imposición y sobreposición de un nuevo

${ }^{4}$ Federal Bureau of Investigation, "Glossary of Terms", EE.uU., Federal Bureau of Investigation (sitio de internet), s/f, https://www.fbi.gov/ investigate/organized-crime\#Glossary-of\%20Terms (consulta del 23 de marzo de 2021).

${ }^{5}$ Enrique Desmond Arias, Criminal Enterprises and Governance in Latin America and the Caribbean, Cambridge, University Press, 2017, pp. 20 y 24-25.

${ }^{6}$ Benjamin Lessing, "Conceptualizing Criminal Governance", Perspectives on Politics, 2020, p. 3. 
orden sobre un territorio que incluye regulaciones económicas y aparatos alternativos de justicia, entre otros factores. La definición de Lessing se construye sobre la idea de que el crimen organizado, más que un actor, es un complejo fenómeno social operando en múltiples dimensiones. En un esquema de gobernanza criminal, los grupos criminales se involucran por igual en la provisión de bienes públicos y en la imposición de reglas y conductas. En síntesis, son una autoridad articulada en torno a un sistema de relaciones que compiten o coexisten con quienes representan al Estado. ${ }^{7}$

Ana Arjona y Nelson Kasfir argumentan que, una vez asegurado el territorio, estos grupos deciden sobre el tipo de interacción con la población. "Pueden robarlos y violarlos, pueden reclutarlos, pueden ignorarlos o pueden tratar de gobernarlos". ${ }^{8}$ Existen diversas combinaciones entre "la creación de una regulación mínima y una tributación informal, hasta la formación de asambleas populares, elaboradas burocracias, escuelas, tribunales y clínicas de salud". ${ }^{9}$ Las variaciones dependen del modelo empresarial de la organización criminal, del tipo de relaciones establecidas con las autoridades gubernamentales, de la personalidad del capo o jefe de plaza y de la resistencia social.

Como insinuamos en párrafos previos, la gobernanza criminal no supone la eliminación o anulación de las autoridades legalmente constituidas. La coexistencia con ellas es muy común, ya sea bajo esquemas de cooperación (facilitados por

${ }^{7}$ Guillermo Trejo y Sandra Ley, Votes, Drugs and Violence:The Political Logic of criminal Wars in Mexico, Cambridge, University Press, 2020; Juan Carlos Garzón, "El futuro del narcotráfico y los traficantes en América Latina”, en Sergio Aguayo y Raúl Benítez (eds.), Atlas de la seguridad y la defensa en México, 2012, México, CASEDE/Open Society, 2012, pp. 101-128; Ioan Grillo, Gangster Warlords. Drug Dollars, Killing Fields and the New Politics of Latin America, Nueva York, Bloomsbury, 2017.

8 Ana Arjona, Nelson Kasfir y Zachariah Mampilly, "Introduction", en Ana Arjona, Nelson Kasfir y Zachariah Mampilly, Rebel Governance in Civil War, Nueva York, Cambridge University Press, 2015, p. 1.

${ }^{9}$ Loc. cit. Todas las citas en este artículo que se encuentran originalmente en idiomas distintos al español han sido traducidas por los autores. 
la corrupción o la complicidad), mediación o subordinación, sobre los cuales flota la posibilidad del uso de las violencias. En otras palabras, la construcción de una autoridad criminal se da de manera paralela y simultánea con la autoridad oficial y legal, o concomitante con la misma autoridad. ${ }^{10}$ En estas situaciones son inevitables las tonalidades de gris. ${ }^{11}$

A continuación explicamos los criterios que nos llevaron a relacionar Chicago, Sicilia y La Laguna. En primer lugar, el hecho de que en los tres existió una gobernanza criminal. En segundo, la participación de los mismos grupos sociales (empresarios, medios de comunicación independientes, colectivos de víctimas, organismos de la sociedad civil, grupos religiosos y académicos) dispuestos a cabildear a representantes del Estado con ideas para contener a los criminales, lo que condujo a la eventual suma de funcionarios comprometidos a luchar contra el grupo criminal local. Finalmente, una reducción posterior sostenible y medible de la criminalidad asociada con la gobernanza criminal.

En investigaciones subsiguientes añadiremos otros centros urbanos (por ejemplo, Nueva York; Medellín, Colombia, y Fortaleza, Brasil) para seguir refinando el modelo aquí desarrollado. También revisaremos la manera en que interactuaron el capital social positivo y negativo en los tres casos, mientras vamos desarrollando una metodología aplicable a urbes como la Ciudad de México.

Lo anterior conduce al concepto de capital social, presente en la literatura de las ciencias sociales desde hace décadas. ${ }^{12}$ Lógicamente, ha ido evolucionando. La idea primigenia es de Pierre Bourdieu, quien lo entendió como los recursos actuales y potenciales que genera una asociación de personas que perdura en el tiempo (que sepamos no hay límites mínip. 93.

${ }^{10}$ Jan Daniel, “Criminal Governance and Insurgency”, CEJISs, 4 (2015),

11 Trejo y Ley, op. cit., p. 9.

12 Ver al respecto el trabajo de Irma Arriagada, "Capital social: potencialidades y limitaciones analíticas de un concepto”, Estudios Sociológicos, XXI, núm. 3 (2003), pp. 557-584. 
mos o máximos de temporalidad) ${ }^{13}$ En sus palabras, el capital social es una "red durable de relaciones más o menos institucionalizadas de reconocimiento y conocimiento -o, en otras palabras, por membresía en un grupo". ${ }^{14}$

Años después, Robert Putnam utilizó el concepto para explicar las razones por las cuales el norte de Italia era más democrático que el sur. Su argumento sorprendió porque demostró que la diferencia estaba en la presencia o ausencia de capital social. ${ }^{15}$ Otros teóricos lo asociaron con las redes sociales. ${ }^{16}$ Es decir, el capital social se expresa y puede estudiarse en términos de redes sociales. Cabe añadir que en esta versión optimista, el capital social se caracteriza por la horizontalidad y la búsqueda de justicia, el respeto a los derechos humanos y la condena de la violencia.

Como sucede con frecuencia, aparecieron aguafiestas como William Callahan, quien sostiene que el "capital social tiene su lado oscuro: grupos muy unidos que trabajan para excluir tanto como incluir". ${ }^{17}$ O como Alejandro Portes, para quien existen consecuencias negativas del capital social. Entre otras, la "exclusión de los no miembros, [las] demandas excesivas a los miembros del grupo [y las] restricciones a la libertad individual". ${ }^{18}$ Los diferenciaremos llamándolos capital social positivo y negativo. Este último se concreta en aquellas relaciones o redes (generalmente

13 Pierre Bourdieu, "The Forms of Capital", en John Richardson (ed.), Handbook of Theory and Research for the Sociology of Education, Nueva York, Greenwood Press, 1986, p. 21.

${ }^{14}$ Loc. cit.

${ }^{15}$ Robert Putnam, Making Democracy Work: Civic Traditions in Modern Italy, Princeton, University Press, (1993).

${ }^{16}$ Mark Granovetter, "The Strength of Weak Ties", American Journal of Sociology, 78, 6, (1973), pp. 1360-1380.

${ }^{17}$ William Callahan, "Social Capital and Corruption: Vote Buying and the Politics of Reform in Thailand", Perspectives on Politics, 3 (3) (2005), p. 495 .

${ }^{18}$ Alejandro Portes, "Capital social: origens e aplicações na sociologia contemporânea”, Revista sociologia, problemas e práticas, 33 (2000), p. 146. 
jerárquicas) que facilitan y sustentan la ilegalidad, la violencia y las relaciones sociales autoritarias.

Si Putnam recurrió a Italia para demostrar lo positivo del capital social, Mauricio Rubio presentó Colombia y Centroamérica como ejemplos de capital social "perverso", al referirse a pandillas basadas en prácticas machistas y masculinidades violentas en general. ${ }^{19}$ Para Heloiza Matos, la forma negativa es "excluyente y crea desconfianza pública, como sucede en el caso de relaciones clientelistas y mafiosas". Siguiendo la lógica de la autora, los contextos de desigualdad en ingresos y de asimetrías de poder son fuentes que propician y acentúan el capital social negativo sobre el cual se asienta el crimen organizado. ${ }^{20}$

A partir de lo anterior, surge la necesidad de categorizar, contextualizar y tipificar el capital social mediante esta dicotomía, en la cual el negativo promueve y fortalece la gobernanza criminal descrita, y el positivo apuntala una base social del Estado de derecho y prácticas de paz capaces de contener la violencia. Por supuesto, no se trata de fórmulas exactas; en este tema, las tonalidades de gris son muy frecuentes.

En las próximas secciones compararemos lo acontecido en Chicago en los años veinte, Sicilia entre los ochenta y los primeros años del siglo xxi, y La Laguna de 2007 a 2014. Pondremos más énfasis en la reacción de los grupos organizados que cabildearon a las autoridades con propuestas específicas que resultaron ser exitosas. Dejamos para otras investigaciones aspectos tan centrales como la forma en que se organizaron, el proceso que los llevó a decidir involucrarse en el combate al crimen organizado y la manera en la que interactuaron con el capital social negativo. También

19 Mauricio Rubio, "Homicidios, justicia, mafias y capital social: otro ensayo sobre la violencia colombiana” (documento), Bogotá, Centro de Estudios sobre Desarrollo Económico, Universidad de los Andes, 1996, http:/ / hdl.handle.net/1992/40933

${ }^{20}$ Heloiza Matos, "O lado escuro do capital social: implicações sociais e políticas”, Líbero, 12 (23), (2009), pp. 56-58. 
posponemos una revisión más detallada sobre la manera en que se forma el capital social negativo.

Aun cuando nos hemos estado refiriendo a seis grupos organizados (empresarios, medios de comunicación, víctimas, académicos, organismos civiles y grupos religiosos), limitaciones de espacio nos obligan a poner más énfasis en los medios (determinantes en la creación de consensos sociales sobre el crimen), en los empresarios y en las víctimas. Los tres son claves en la definición del problema y en la interlocución con las autoridades.

Ordenaremos la evolución en tres etapas: la coexistencia entre el grupo criminal y la aún incipiente respuesta social, el punto de quiebre asociado a un hecho particularmente violento y, por último, la aplicación de estrategias públicas consensuadas entre Estado y sociedad civil con un final exitoso que se sostiene en el tiempo. En ningún caso se eliminó por completo la presencia criminal, pero sí hubo una disminución de la violencia a niveles aceptables para los grupos sociales y el gobierno.

\section{LA COEXISTENCIA}

Insistimos en el patrón: en los tres casos, primero surgió el grupo criminal y luego vino la reacción en contra de éste. Empezamos por la primera etapa: la coexistencia, misma que bosquejaremos caso por caso.

\section{Chicago}

Una ventaja de empezar con Chicago es que la presencia del crimen organizado en los años veinte ha sido muy estudiada y eso permite apreciar con nitidez las etapas de coexistencia, quiebre y ataque a las bandas.

Los estudiosos coinciden en el peso de la Prohibición (1920-1933) tras la aparición del crimen organizado. Chicago 
era la segunda urbe de Estados Unidos y la mayoría de su población se oponía a la ley seca (en la jerga de la época era una ciudad "mojada" -wet-). Esto facilitó la aceptación o tolerancia de los gangsters. Aun cuando había diferentes grupos criminales, la que atraía los reflectores era la organización de Al Capone. Es lógico que así fuera debido a la dimensión del grupo y al carisma de Capone, quien se presentaba ante la prensa como un empresario y "benefactor" que suministraba a la sociedad "placeres menores" (alcohol, prostitución y juego).$^{21}$

Para Eliot Ness, Capone "poseía el genio organizativo" que lo hizo "convertirse en el zar del crimen" de Chicago. ${ }^{22}$ En 2010, la Escuela de Negocios de Harvard recurrió a la organización de Capone para hacer uno de sus famosos estudios de caso. Para 1929, su banda obtenía 100 millones de dólares de ingresos anuales (1490 mdd a diciembre de 2019); de éstos, 60 millones provenían de cerveza y licores, 25 del juego, 10 de la prostitución y los bares, y 10 eran ingresos misceláneos. Sintetizamos la estructura organizativa porque es un modelo que todavía reproducen otras organizaciones criminales. Tenía tres grandes ramas:

a) Para administrar las diversas empresas, la organización contaba con gerentes, tesoreros, contadores y responsables de las ventas de licor. Dos personas se encargaban de administrar el centenar de burdeles propiedad de la familia Capone. Estarían después los encargados de los salones de juego y así sucesivamente.

b) La violencia era esencial y la organización tenía golpeadores, asesinos y especialistas en bombas. Los entrenaba y capacitaba para hacer bien su peculiar oficio. Por ejemplo, a los competidores que osaban vender

21 Daniel McDonough, "Chicago Press Treatment of the Gangster, 1924-1931”, Illinois Historical Journal, vol. 82, núm. 1, (primavera de 1989), pp. 17-32, https://www.jstor.org/stable/40192250

${ }^{22}$ Laurence Bergreen, Capone: The Man and the Era, Nueva York, Simon and Schuster (Kindle edition), 1996, p. 508. 
cerveza en sus territorios, les destruían sus negocios y con frecuencia los ejecutaban.

c) Por último, un departamento legal se encargaba de sacarlos de la cárcel, además de otros apoyos acordes con el nivel de vida e imagen del capo: secretarios, choferes, doctores, valets, barberos, chefs, meseros, etcétera. ${ }^{23}$

La relación de la organización con la autoridad se basaba en el reparto de dinero a cambio de tolerancia y protección. Dedicaba una tercera parte de los ingresos al soborno de políticos y policías estatales y municipales (se estima que tenía en su nómina a la mitad de los policías de la ciudad de Chicago). La infiltración se facilitaba porque por aquellos años el gobierno federal no perseguía al crimen organizado.

Las organizaciones criminales tenían una fuerte base social en los barrios pobres. De ahí provenían sus asesinos y buena parte del elaborado sistema de inteligencia que vigilaba toda la ciudad para la organización. Tenían a su servicio a un alto porcentaje de meseros, prostitutas, taxistas y voceadores. Al Capone dirigía una organización muy bien informada.

Conocemos otros rasgos de las mafias de Chicago por la obra de John Landesco, un académico visionario cuyo libro principal (El crimen organizado en Chicago, de 1929) ha sido injustamente olvidado. "El gangster," escribió Landesco, "es un producto de su entorno al igual que el buen ciudadano es resultado de su ambiente". ${ }^{24}$ En su estudio también explica el impacto de la prohibición. Antes de esa ley -considera- había "relaciones de amistad" entre criminales y policías porque ambos provenían de los mismos barrios. Cuando transportar, comerciar o consumir bebidas alcohólicas se vuelve ilegal, la relación pasa a basarse en el dinero. ${ }^{25}$ La corrupción se profesionaliza.

23 Tom Nicholas y David Chen, Case 9-809-144. Al Capone, Harvard Business School, marzo de 2012.

${ }^{24}$ John Landesco, Organized Crime in Chicago. Part III of the Illinois Crime Survey, 1929, Chicago, The University of Chicago Press, 1968, xi.

${ }^{25}$ Ibid., xii. 
Capone dedicaba tiempo y recursos a las relaciones públicas y a la promoción de su imagen. Amaba los reflectores y la ostentación de sus riquezas. Iba a las peleas de "box, al hipódromo o a la ópera" con "18 guardaespaldas". Durante sus primeros años, los principales diarios de la ciudad (The Chicago Tribune y el Chicago Herald and Examiner) lo trataron como una celebridad y tenían a reporteros cubriendo "sus actividades". ${ }^{26}$ Los turistas deambulaban frente al hotel donde vivía con la esperanza de contemplarlo para poder relatar, en sus lugares de origen, el avistamiento de un personaje de fama internacional. ${ }^{27}$ Cuando inició la Gran Depresión en 1929, cultivó su imagen filantrópica instalando comedores populares para los pobres. Era objeto de fascinación pública.

\section{Sicilia}

La Cosa Nostra, en Sicilia, ya existía antes del Risorgimento (surgimiento del Estado italiano moderno en 1861). Uno de los mitos sobre su origen es la llegada a Italia de tres caballeros españoles con apellidos que riman (Osso, Mastrosso y Carcagnosso). Desembarcaron en Sicilia. Osso se quedó ahí para fundar la Cosa Nostra. Mastrosso lo emularía en Calabria con la 'Ndranghetta, y Carcagnosso en Nápoles, con la Camorra. ${ }^{28}$

La Cosa Nostra siciliana logró arraigarse en el territorio, la sociedad y la cultura. Estableció una gobernanza criminal que dominaba valiéndose de la violencia y el convencimiento. ${ }^{29}$ En los años ochenta del siglo xx, el primer arrepentido (pentito), Tommaso Buscetta, confirmó que la estructura de

${ }^{26}$ McDonough, art. cit., p. 29.

27 Ken Burns y Lynn Novick (dirs.), "Una nación de hipócritas", Prohibition, cap. 3 (serie documental), Netflix, 2011.

28 John Dickie, Historia de la mafia. Cosa nostra, 'Ndrangheta, Camorra de 1860 al presente, Barcelona, Penguin Random House, 2015.

${ }^{29}$ Anton Blok, The Mafia of a Sicilian village, 1860-1960: A study of violent peasant entrepreneurs, Londres, Harper and Row, 1974. 
la Cosa Nostra estaba construida sobre el respeto a la jerarquía, el apego a los lazos familiares ${ }^{30}$ y la lealtad a la organización que tenía su máxima expresión en el silencio (la omertá). ${ }^{31}$

Para Umberto Santino, ${ }^{32}$ la relación de la Cosa Nostra con el Estado y en general con la autoridad legal tiene dos dimensiones: como asociación delictiva y como gobernanza alternativa en el sentido weberiano. Es decir, la organización contaba con:

a) Un conjunto de reglas (ordenamiento).

b) Una dimensión territorial.

c) La posibilidad de utilizar la coerción física.

d) Un aparato administrativo capaz de hacer cumplir las normas. (A continuación, ampliamos este inciso crucial).

Las relaciones de la Cosa Nostra con la sociedad se construían sobre una combinación de intimidación, subordinación y convencimiento. El grupo criminal impuso, con el miedo, una cohesión interna suficientemente amplia y consolidada como para establecerse territorialmente en toda la isla y esa cohesión se presentó como resultado de una autoridad local válida, capaz de ejercer el poder, controlar y administrar la violencia, actividades económicas y comerciales, así como códigos de conducta basados en la autoridad reconocida de la mafia. Finalmente, una parte de la sociedad estaba convencida de la legitimidad de esa gobernanza criminal.

El punto cumbre del poderío de la Cosa Nostra se dio a finales de los años setenta y principios de los ochenta. Para ese momento, había establecido poderío e impunidad abrumadoras basado en el control de las autoridades legales. Tam-

30 Adela Garzón, "Familismo y creencias políticas", Psicología Política, núm. 17, (noviembre de 1998), pp. 101-128.

${ }^{31}$ Jane Schneider y Peter Schneider, "Mafia, Antimafia, and the Plural Cultures of Sicily", Current Anthropology, vol. 4 (agosto-octubre de 2005), pp. 501-520.

32 Umberto Santino, Breve Storia della Mafia e dell'Antimafia, Palermo, Di Girolamo, 2011, pp. 39-40. 
bién había logrado esparcir entre la sociedad el miedo a su mandato criminal. En suma, proyectaba una imagen de omnipotencia y omnipresencia.

\section{La Laguna}

La coexistencia se expresó de diferente manera en La Laguna, una ciudad compartida entre los estados norteños de Coahuila y Durango, en México. Esta zona tiene una gran importancia estratégica para el tráfico de drogas por ser el principal nodo de comunicación entre el Pacífico y la frontera del noreste mexicano con Estados Unidos. Para viajar por tierra de Culiacán a Ciudad Juárez, Nuevo Laredo y Reynosa se pasa por La Laguna. Es la vía más corta. Por tanto, la sociedad se acostumbró a la presencia criminal desde los años veinte del siglo pasado.

Durante mucho tiempo, el cartel de Juárez dominó ese territorio. Cuando se disolvió la alianza de Juárez con el cartel de Sinaloa en los primeros años del siglo xxi, Sinaloa se convirtió en la organización dominante. La sociedad los toleraba porque había el supuesto implícito de que "ellos" vivían separados, en un mundo aparte.

La llegada de los Zetas alteró el equilibrio. Este grupo se distinguía por la brutalidad sistémica. Recurrían a niveles de violencia poco habituales: desmembramientos, decapitaciones y disolución de restos humanos en ácido. También establecieron campos de entrenamiento para capacitar a sus sicarios en el uso de todo tipo de armas, en tácticas militares de combate, tortura, técnicas de contrainteligencia y telecomunicaciones. Los fundadores de la organización difundían la adaptación de lo aprendido en Estados Unidos durante su formación como tropas de elite.

Los Zetas llegaron a La Laguna entre 2003 y 2004. Durante dos o tres años mantuvieron un bajo perfil y fueron sentando las bases para controlar la plaza con una cadencia ensayada por los criminales en otras regiones. El primer paso 
fue el reclutamiento de elementos de diferentes corporaciones policiacas y unidades militares. Utilizaban a los agentes encargados de aplicar la violencia de forma legítima para allegarse información, capturar personas, cobrar "derecho de piso" y controlar el territorio.

Una vez infiltradas las instituciones de seguridad, los Zetas empezaban a intimidar a empresarios, medios de comunicación y sociedad, valiéndose del mismo método. Primero, reclutaban a integrantes del gremio respectivo para usarlos como informantes y mensajeros. Una vez implantados, los Zetas impartían una cátedra de violencia pedagógica. Secuestraban y ejecutaban a algún integrante del gremio respectivo para infundir miedo y exigir obediencia. ${ }^{33}$ Ésta fue la lógica tras el atentado contra el empresario Carlos Herrera en 2007 y el asesinato del periodista Eliseo Barrón. Para aterrorizar a la sociedad, esparcían partes humanas por la ciudad y perpetraban matanzas en lugares concurridos.

La fuerza lograda por los Zetas sobre La Laguna llegó a ser tan opresiva, que se gestó la resistencia de varios grupos sociales. Como veremos en la siguiente sección, en eso influyó una matanza en Tamaulipas que modificó la estrategia federal.

\section{EL PUNTO DE QUIEBRE}

Encontramos como patrón la existencia de un límite en la tolerancia social y gubernamental a la violencia criminal. Es un umbral variable, pero cuando se cruza, da lugar a la reacción de alguno o varios de los actores sociales ya mencionados. Empieza entonces la búsqueda de soluciones y el acercamiento a las autoridades con peticiones concretas para reducir la gobernanza criminal a niveles tolerables. Otro patrón observable es la aceptación tácita de sociedad y gobier-

${ }^{33}$ Guadalupe Correa-Cabrera, Los Zetas Inc. La corporación delictiva que funciona como empresa transnacional, México, Planeta, 2018. 
no sobre la imposibilidad de extirpar por completo al crimen organizado. Revisemos en más detalle los tres casos.

\section{Chicago}

En 1924 dio inicio en Chicago la "guerra de la cerveza". Grupos criminales antagónicos se enfrentaron a balazos y bombazos para controlar el lucrativo negocio. A partir de ese año, explica Daniel McDonough, cambió la opinión de una parte de la prensa. Capone empezó a ser visto como el jefe de una "fuerza despiadada, viciosa y siniestra". ${ }^{34}$ Empezó su demonización y Capone pasó de ser el empresario benefactor al "supervillano de aquella época”. ${ }^{35}$

Capone y su banda habían suplantado al gobierno del condado de Cicero (conurbado con Chicago), ciudad en donde apareció en 1924 el The Cicero Tribune. Lo fundó Robert St. John a los 21 años, con una línea editorial centrada en la denuncia sistemática y metódica de las tropelías de Capone y sus gatilleros, la protección otorgada por la autoridad y la indefensión de la ciudadanía.

Capone reaccionó ordenando una golpiza tan brutal que St. John estuvo en el hospital durante una semana. No se arredró. Siguió adelante, pero el capo lo derrotó con los métodos de la economía de mercado: compró la mayoría de acciones del The Cicero Tribune. El periodista se rindió y dejó Cicero para hacer una carrera brillante como corresponsal de guerra. ${ }^{36}$ Murió a los 100 años de edad sin regresar jamás a la ciudad donde empezara su carrera. Pese a su derrota, dice el biógrafo de Capone, St. John hizo "más para atacar a los gangsters y sus perversas tácticas que toda la policía y los políticos de Chicago". ${ }^{37}$ Otros periodistas siguieron su ejemplo y

\footnotetext{
34 McDonough, art. cit., p. 29.

35 Bergreen, op. cit., p. 530.

36 Ibid., pp. 122-123.

37 Ibid., p. 118.
} 
fueron creando las condiciones para un cambio de actitudes que desembocaría en el ocaso de Capone.

En los años siguientes la violencia no amainaba y el momento del quiebre vino con el asesinato de siete personas el Día de San Valentín, en 1929. Aquel 14 de febrero, un grupo de gangsters vestidos como policías llegó al local de un enemigo de Capone. Cuando vieron los uniformes, seis pistoleros y un inocente que estaba por accidente en el lugar, obedecieron la orden de ponerse contra la pared. Fueron ametrallados. El escándalo fue nacional y desbordó la paciencia de la sociedad organizada, que tenía años denunciando la presencia criminal.

Incluso los capos de las familias de otras ciudades estadounidenses estaban cansados de la excesiva violencia de Chicago. Era nociva para los negocios. Después de visitar la ciudad, el famoso gangster neoyorquino, Charles "Lucky" Luciano la describió como "un lugar endiabladamente loco. Nadie está seguro en las calles". ${ }^{38}$ Después de aquel Día de San Valentín, los jefes de la delincuencia neoyorquina se reunieron -en mayo de 1929- y concluyeron que la violencia estaba afectando los negocios de todos; era "necesario sacrificar un cordero para reducir la presión. Y Capone sería ese cordero". 39

Un mes después de la masacre del Día de San Valentín, un grupo de empresarios y líderes cívicos de Chicago viajaron a Washington para entrevistarse con el presidente Herbert Hoover. "Capone gobierna la ciudad", se quejaron. Hoover reaccionó "orden[ando] a todas las agencias federales concentrarse en el señor Capone". Debían "meterlo en la cárcel". ${ }^{40}$ La decisión presidencial fue importante porque hasta ese momento el gobierno federal no combatía al cri-

${ }^{38}$ Ibid. p. 908.

${ }^{39}$ Martin A. Gosch y Richard Hammer, The Last Testament of Lucky Luciano: The Mafia Story in His Own Words, Enigma Books (edición Kindle), 1975, posiciones, 2033-2034.

${ }^{40}$ Arthur M. Schlesinger, Robert Kennedy and his Times, Boston y Nueva York, Houghton Mifflin, 2018, p. 284. 
men organizado, debido a que le correspondía hacerlo a las autoridades municipales y estatales. Fue una intervención excepcional. Pasarían muchos años para que se declararan competentes en la materia.

\section{Sicilia}

El arraigo centenario de la mafia siciliana se consolidó después de la Segunda Guerra Mundial. La Guerra Fría cambió las prioridades de Washington que, para frenar el avance comunista en Europa, empoderó a las mafias italianas y francesas. "En pueblo tras pueblo italiano, los aliados nombraron alcaldes a personajes ligados a la mafia"; la prioridad era evitar el "nombramiento de comunistas". ${ }^{41}$ En otras palabras, por el "anticomunismo de la posguerra, Estados Unidos hizo un trato con el diablo". Su prioridad era defender la democracia liberal y la economía de mercado en el mundo, y desprotegió a los adictos estadounidenses que empezaron a recibir drogas de Sicilia y Marsella. ${ }^{42}$

Con este antecedente, veamos las dos etapas del movimiento antimafia siciliano. En la primera se le asociaba con la izquierda representada por el Partido Comunista Italiano. En la segunda, el movimiento se volvió plural a partir del surgimiento de los "cadáveres excelentes", término con el cual se identificó a las víctimas emblemáticas de la mafia. ${ }^{43}$ Una vez más, la violencia generó una reacción en contra.

Entre los "cadáveres excelentes" hay periodistas, sacerdotes, funcionarios públicos, militares, empresarios e intelec-

${ }^{41}$ Gil Reavill, Mafia Summit: J. Edgar Hoover, the Kennedy Brothers and the Meeting that Unmasked the Mob, Nueva York, Thomas Dunne Books (edición Kindle), 2015.

42 Loc. cit.

43 Jane Schneider y Peter Schneider, "Civil Society Versus Organized Crime”, Critique of Anthropology, 21, 4 (2001), p. 431; Letizia Paoli, Mafia Brotherhoods: Organized Crime, Italian Style, Oxford, University Press, 2003, p. 12. 
tuales y, finalmente, jueces antimafia. De alguna manera, la heterogeneidad de los casos alimentó la pluralidad de una causa que, a la postre, se articuló en torno a dos ejes: el rechazo del poder de la mafia y la disputa del orden social promovido por la Cosa Nostra. A continuación mencionamos los casos más relevantes.

Giuseppe "Peppino" Impastato, periodista y activista local. Nacido en familia mafiosa, rompió con ellos para volverse comunista. En 1977 creó Radio Aut, desde donde condenaba públicamente las actividades de la mafia, que respondió con una ejecución pedagógica espectacular: ataron al periodista a las vías del tren y luego lo dinamitaron. Querían educar a la población sobre lo que sucedía a quienes retaban su gobernanza. Su muerte motivó a la madre y al hermano, entre otros, para investigar el crimen y demostrar la responsabilidad mafiosa. ${ }^{44}$ Sus esfuerzos llevaron a la creación del Centro Impastato, una importante institución dedicada a la documentación sobre la mafia.

Carlo Alberto dalla Chiesa, General de División de los Carabinieri. En 1982, Dalla Chiesa se convirtió en prefecto de Palermo y coordinador de una campaña nacional contra la mafia. Duró en el cargo cuatro meses, antes de ser ejecutado a balazos junto a su esposa. Como su nombramiento simbolizaba "el deseo del Estado de presentar una postura pública fuerte contra la mafia", el asesinato confirmó la "falta de respeto" criminal a las instituciones gubernamentales. ${ }^{45}$

Giuseppe "Pino" Puglisi, sacerdote católico. Estableció una pastoral antimafia en Brancaccio, un barrio de Palermo con fuerte presencia de la mafia. Su trabajo más visible lo hizo con

${ }^{44}$ Federico Varese, Vita di mafia. Amore, morte e denaro nel cuore del crimine organizzato, Torino, Giulio Einaudi editore, 2017, p. 207.

45 Jane Schneider y Peter Schneider, Reversible Destiny. Mafia, Antimafia, and the Struggle for Palermo, California, University of California Press, 2003, p. 100. 
niños en situación de calle, a quienes presentaba una imagen alternativa de los mafiosos, considerados modelos de vida por la conveniencia. ${ }^{46} \mathrm{El}$ sacerdote también criticaba a la mafia en los sermones. Pino tenía 56 años cuando lo asesinó un sicario (el término es siciliano). Se dice que al verlo pronunció, como últimas palabras, "te estaba esperando". Esa disposición al martirio probablemente influyó para su beatificación en 2013 por el papa Francisco. Su tumba se encuentra en la catedral de Palermo. Es un símbolo para los católicos antimafiosos.

Libero Grassi, empresario. Después de recibir amenazas como tantos otros comerciantes y empresarios locales, Grassi se negó a pagar el pizzo (extorsión) e hizo pública su negativa en una carta dirigida a su Caro estortore (estimado extorsionador), que apareció en la prensa local. Su asesinato provocó uno de los más exitosos modelos de articulación entre autoridades y organizaciones de la sociedad civil.

Giovanni Falcone y Paolo Borsellino, jueces antimafia. Ambos son, probablemente, el símbolo más importante de la antimafia y por tanto, 1992 sería el momento simbólico de su quiebre. Falcone y Borsellino lideraron el primer y emblemático pool antimafia siciliano, es decir, el conjunto de magistrados ocupados de manera coordinada y colectiva de investigar criminalmente a la Cosa Nostra. Su mayor logro fue el Maxiprocesso, un juicio sin precedentes en Europa por su magnitud (lo discutiremos en la tercera sección). Fueron asesinados en un atentado espectacular de la mafia en mayo y julio de 1992, respectivamente, y se convirtieron en un símbolo que cohesiona a casi todos los sectores antimafia en torno a una tesis: la violencia mafiosa y el sistema que la hacía posible rebasaba los límites tolerables. Debía ser combatida.

${ }^{46}$ Marco Corvaia, Pino se lo aspettava. Il racconto de la vida e della morte di Padre Puglisi, Palermo, Navarra Editore, 2012, p. 60. 


\section{La Laguna}

Lo ocurrido en esta región tiene similitudes y diferencias con Chicago y Sicilia. Es parecida por la participación de empresarios, víctimas y medios y por la manera en que interactuaron hechos locales y nacionales. Es diferente por el peso que tuvo el enfrentamiento entre dos carteles y por el impacto nacional e internacional de una matanza en un estado vecino.

Como veremos a continuación, los laguneros se movilizaron cuando se daba un cambio radical en la estrategia del gobierno federal y estatal. En 2009, comenta Guillermo Valdés -en aquel momento director del Centro de Investigación y Seguridad Nacional-, "nos dimos cuenta de que no podíamos ganar la guerra” contra todas las organizaciones criminales. "En consecuencia, cambiamos el objetivo a la reducción de la violencia y decidimos irnos contra los Zetas". ${ }^{47}$

La decisión se aceleró por un hecho de particular brutalidad. La matanza de 72 migrantes en San Fernando (agosto de 2010) fue un punto de quiebre. Se redefinió el objetivo del gobierno -continuó Valdés-y se puso en primer lugar "garantizar la paz y la seguridad de la población". Se trataba de "reducir violencia en lugar de la destrucción del narcotráfico o el combate abierto a las organizaciones". Valdés Castellanos presumía que "en menos de tres semanas teníamos como al 90\% de los involucrados en la masacre de San Fernando". ${ }^{4}$

El cartel de Sinaloa tenía décadas de estar asentado en La Laguna. Había logrado un entendimiento tácito con la población: traficaban con drogas, es cierto, pero cuidaban de no molestar a los civiles. La situación cambió con la llegada de los Zetas y su modelo de negocios depredador y extremadamente violento. El choque entre ambos grupos abrió espacios para que algunos sectores de la sociedad empezaran a preguntarse qué podrían hacer para recuperar la paz.

47 Conversación con Guillermo Valdés durante un seminario académico realizado en la Universidad de Oxford el 1 de noviembre de 2019.

${ }^{48}$ Loc. cit. 
Una advertencia: esta sección será diferente a las anteriores porque reduciremos el énfasis en los hechos de violencia, para poner el acento en la respuesta social a partir de un intangible: la identidad colectiva de los laguneros. Javier Garza -prestigiado periodista- la definió como una "identidad de independencia, un sentimiento de que las cosas se ganan con trabajo". También influyó un "sentido de solidaridad, y de ayuda mutua". 49

\section{Empresarios}

La movilización de empresarios empezó en la capital del país cuando, en 2008-2009, un grupo encabezado por Alejandro Martí (padre de una víctima de secuestro) se organizó para trabajar por la seguridad en todo el país. Una de las primeras medidas fue crear la organización México Evalúa en 2009. Su actual directora, Edna Jaime, relata que su "primera tarea fue generar un sistema de indicadores para dar seguimiento al fenómeno delictivo" creando observatorios para "orientar a la opinión pública mexicana". Un rasgo distintivo de estos observatorios fue el rechazo de financiamientos públicos. ${ }^{50}$

Los empresarios de La Laguna se distinguían por su independencia y fue uno de los primeros lugares en crear un observatorio. Edna Jaime lo adjudica a que "son empresarios de alguna manera globalizados, que no dependen de su relación con el gobernador. Necesitan del gobierno local o del gobierno estatal para poder funcionar, necesitan que haya provisión de servicios públicos básicos, pero no le deben más allá de eso. No hay rentismo". ${ }^{51}$

${ }^{49}$ Comunicación personal del periodista Javier Garza Ramos (correo electrónico), exsubdirector editorial de El Siglo de Torreón, 8 de diciembre de 2019.

${ }^{50}$ Sergio Aguayo y Jacobo Dayán, "Reconquistando" La Laguna, México, El Colegio de México, 2020, p. 68.

${ }^{51}$ Loc. cit. 
La primera medida tomada por los empresarios locales fue crear el Consejo Cívico de las Instituciones Laguna organización que elaboró "indicadores que van más allá de la incidencia delictiva. Se convirtieron en los más innovadores de todos los Observatorios”. Entre otros aspectos, Edna Jaime menciona que "empezaron a georreferenciar los delitos, a construir mapas de calor, a elaborar puntos calientes". Actualmente "es un observatorio que además de temas de seguridad monitorea el gasto y las obras públicas". Es una institución que "pide cuentas a la autoridad" y la vigila. ${ }^{52}$

De manera simultánea, un grupo de grandes empresarios empezaron a cabildear con los gobiernos federal, estatal y municipal. En 2011 se reunieron con Jorge Tello Peón, asesor del presidente Felipe Calderón y cercano a los empresarios de Monterrey. Tello relata: "En la segunda mitad de 2011 se hizo una primera reunión en la casa de uno de ellos. Invitaron a los dos gobernadores; yo diría a los tres, porque el gobernador electo era Rubén Moreira pero estaba de interino Jorge Torres y acababa de tomar posesión Jorge Herrera de Durango. Se les planteó el proyecto conjunto”. ${ }^{53}$ Más adelante abordaremos las peticiones que hicieron y las respuestas que recibieron.

\section{Los colectivos de víctimas}

En 2009 se creó en Saltillo -capital de Coahuila- Fuerzas Unidas por Nuestros Desaparecidos en Coahuila (Fuundec). Instalaron una oficina en La Laguna y tuvieron el apoyo de la Diócesis de Saltillo encabezada por el obispo Raúl Vera.

Es frecuente que los colectivos (o colectivas como prefieren llamarse) surjan a partir de una iniciativa individual. En noviembre de 2004 desapareció Silvia Stephanie Sánchez-

${ }^{52}$ Loc. cit.

${ }^{53}$ Entrevista de Sergio Aguayo y Jacobo Dayán a Jorge Tello, director de Madison Inteligencia, 19 de noviembre de 2019. 
Viesca Ortiz. Su familia, encabezada por la madre, Silvia Ortiz, se movilizó de inmediato y se puso a pegar carteles, realizar marchas, organizar misas y protestas. En los primeros años los acompañaron familiares y amigos. Luego se fueron quedando solos pero, como insiste doña Silvia, "fuimos tercos y tesoneros". ${ }^{54}$ Levantaban denuncias pese a las trabas que les ponían y daban entrevistas a medios. Sus principales aliados eran los medios de comunicación y los grupos religiosos, mientras que los empresarios los ignoraban. En 2009 crearon el Grupo Vida.

Esta iniciativa coincidió con la aparición del Movimiento por la Paz con Justicia y Dignidad (MPJD) en la capital del país. Se trataba de una movilización nacional encabezada por el poeta Javier Sicilia, padre de un joven asesinado en marzo de 2011. Una de las caravanas llegó a Torreón, Coahuila, en junio de 2011 y empezaron a interactuar los eventos nacionales y locales. El Grupo Vida, por ejemplo, emuló a los padres de los normalistas de Ayotzinapa y salió al campo a buscar, ellos mismos, los restos de desaparecidos. Comenzaron en enero de 2015 y, hasta mayo de 2019, habían logrado identificar 24 sitios con restos óseos.

\section{Periodistas}

Los periodistas que decidieron resistir los ataques criminales partieron siempre de una premisa: no podían contar con el apoyo gubernamental. Se generaron, entonces, procesos espontáneos a partir de las medidas de protección que adoptaba cada periodista.

La solidaridad gremial y la unidad se dio en torno a un objetivo: sobrevivir e informar hasta donde fuera posible. En un gremio caracterizado por los protagonismos, fue notable la claridad que tuvieron para hacerlos a un lado. Una de sus primeras medidas fue socializar la información; nadie

${ }^{54}$ Entrevista a Óscar Sánchez Viesca, 14 de mayo de 2019. 
iba solo a los lugares de los enfrentamientos y estaban en comunicación permanente. Armando Moreno recuerda al respecto: "Aprendí que la muerte se tiene que respetar, que no hay egos". 55

Los periodistas se volvieron extremadamente cuidadosos con el lenguaje. Utilizaban palabras neutras: traviesos, malos, aquellos, "los de la compañía", etcétera. También observaban con cuidado los códigos no escritos de los criminales y sus pautas de comportamiento. Estaría, finalmente, una fórmula pragmática que sintetiza Javier Garza, entonces director editorial de El Siglo de Torreón: "Un punto de consenso entre los periodistas era que sólo publicarían aquello que fuera confirmado por las autoridades por medio de un boletín, parte o rueda de prensa" ${ }^{56}$ En otras palabras, si el gobierno estaba infiltrado entonces la información difundida por un funcionario sería aceptable para "ellos". También modificaron el contenido de las notas. Por aquellos años, $E l$ Siglo de Torreón:

[...] adoptó una forma muy limitada y básica de informar sobre el crimen organizado. Nos apegamos a la información oficial sin conducir nuestras investigaciones. Lo compensamos con historias que incluían estadísticas criminales, el aumento de los robos armados, el impacto económico y social de la violencia, los testimonios de quienes vivían a su sombra y los vínculos entre la pobreza y el desempleo con el crimen. ${ }^{57}$

Estaría, finalmente, el respaldo que buscaron y obtuvieron de medios nacionales e internacionales y de organizaciones de protección a periodistas. Al trascender el ámbito

${ }^{55}$ Entrevista de Francisco Rodríguez a Armando Moreno, Milenio Laguna, 13 de mayo de 2019.

${ }^{56}$ Entrevista Javier Garza Ramos, exsubdirector editorial de El Siglo de Torreón. 9 de noviembre de 2017.

${ }^{57}$ Javier Garza Ramos, "Under Fire in Mexico", Harvard Review of Latin America, Journalism of the Americas (primavera-verano de 2011), https:// revista.drclas.harvard.edu/book/under-fire-mexico 
local, ampliaron los márgenes de protección y hubo mayor presión sobre los tres niveles de gobierno para que pusieran en la lista de prioridades el combate al cártel más peligroso: los Zetas.

\section{LAS ESTRATEGIAS CONSENSUADAS}

$\mathrm{El}$ acuerdo entre sociedad y gobierno en una estrategia requería, antes que nada, de que independientemente de la manera en que las partes definieran el riesgo, coincidieran en la urgencia de frenar a los criminales. A partir de ahí venía la convergencia en medidas concretas que variaban en los niveles de conciencia sobre el problema y las características de cada cultura y sistema político y legal. La meta era muy clara: reducir la violencia a límites tolerables aceptando, tácitamente, la imposibilidad de erradicar totalmente al crimen organizado.

\section{Chicago}

Como se mencionó antes, tras la masacre del Día de San Valentín en 1929, el presidente Herbert Hoover ordenó el encarcelamiento de Capone, con lo cual dio inicio la participación del gobierno federal en el combate al crimen organizado.

Dada la negativa del director de la Oficina de Investigación (posteriormente sería el FBI) a reconocer la existencia de la mafia, el presidente encomendó la tarea al Departamento del Tesoro, que tomó dos rutas paralelas. El Servicio de Impuestos Internos (IRS) averiguaría si Capone había pagado impuestos y la Oficina de la Prohibición (Bureau of Prohibition) investigaría las violaciones cometidas a la ley seca. En la primera estaban los contadores, en la segunda, Eliot Ness y "un grupo pequeño e incorruptible", que se ha- 
ría famoso por su apodo de "los intocables" y sería inmortalizado por la televisión y el cine. ${ }^{58}$

En diciembre de 1930, Ness ya había creado un equipo, muy pequeño (nunca superó la decena de varones de raza blanca), para atacar una organización dedicada al trasiego de licores, a la extorsión, al control de sindicatos, a la prostitución y al juego. Ness tenía órdenes de documentar delitos y atacar las fuentes de ingreso de Capone. Optó por ir contra las cervecerías clandestinas, porque tenían la "mayor inversión de capital, el ingreso más grande y las ganancias más rápidas". ${ }^{59}$

Para cumplir con su encomienda se apoyó en la inteligencia (información procesada) y para ello hicieron un listado con todos los gangsters de la región, ordenó una vigilancia permanente de los principales bares clandestinos ("speakeasies") e intervino los teléfonos de las habitaciones del hotel donde vivía Capone y de sus casinos y prostíbulos. Recibió asesoría del Laboratorio Forense del Crimen de la Universidad Northwestern de Chicago, financiado por un grupo semiclandestino de empresarios (los Secret Six).

En la primavera de 1931, el fiscal federal tenía sobre su escritorio material para acusar a Capone de violar la ley seca (trabajo de Ness y sus "intocables") o de evadir impuestos por un millón de dólares (14.5 millones de dólares en diciembre de 2018) de ingresos obtenidos entre 1924 y 1929. El 5 de junio optó por la segunda alternativa. Un jurado de ciudadanos afectados por la Gran Depresión -razonó el fiscal- estaría más dispuesto a condenar a Capone por no querer pagar impuestos. El caso armado por Ness y su equipo se dejó en reserva por si fallaba el de los contadores. Nunca lo utilizaron. En octubre de 1931, Capone fue condenado a 11 años de prisión por el prosaico delito de no pagar los impuestos que debía. Gracias a Hollywood y a los medios, los laureles y medallas se las colgaron a Ness y sus "intocables". ${ }^{60}$

58 Douglas Perry, Eliot Ness: The Rise and Fall of an American Hero, EE.UU., Viking (edición Kindle), 2014, 337 pp.

${ }^{59}$ Loc. cit.

${ }^{60}$ Loc. cit. 
Sin embargo, las autoridades se equivocaban al creer que encarcelando a Capone terminarían con el crimen organizado. Ese mismo año se reunieron en un hotel de Chicago los padrinos de siete familias: cinco de Nueva York, una de Chicago y una de Búfalo. En esa cumbre, presidida por "Lucky" Luciano, crearon "la Comisión", una junta directiva encargada de trazar las grandes líneas estratégicas, resolver los conflictos entre mafiosos de manera pacífica y repartirse los mercados del país entero. Tuvieron que pasar décadas para que gobierno y sociedad de Estados Unidos aceptaran la existencia de una organización criminal nacional y diseñaran las estrategias y leyes para combatirla.

Un momento que simboliza el éxito fue el "Macrojuicio de 1986", cuando juzgaron a los jefes de las cinco familias neoyorquinas. Lo relevante de ese juicio es que estuvo pensado para reducir el poder de los criminales asumiendo, de entrada, la imposibilidad de erradicarlos. El segundo del fiscal Rudolph Giuliani, Michael Chertoff, tuvo un arrebato de claridad cuando aclaró que el Macrojuicio fue "diseñado en su totalidad para quebrar el poder económico de las organizaciones criminales y regresarlas a sus orígenes, cuando eran simplemente pandillas". ${ }^{61}$ Les interesaba reducir la gobernanza criminal a niveles aceptables. Dada su complejidad, dejamos para otro texto la lógica detrás de la autolimitación.

\section{Sicilia}

Por una de esas coincidencias que tiene la historia, el Macrojuicio de Nueva York se empalmó con el "Maxiprocesso" siciliano, entre 1985 y 1986. El Maxiprocesso logró reunir pruebas legales contra mafiosos gracias en buena medida a las declaraciones de Tommaso Buscetta, el primer pentito (arrepentido).

61 "Taking Down the Mob" (cap. 4), Inside the American Mob (serie documental), National Geographic, 2013, minutos 34:28 a 34:37. 
Con la información aportada por Buscetta, el juez Falcone terminó de entender la estructura de la Cosa Nostra.

Liderado por Falcone, el pool antimafia identificó a los miembros de la Cosa Nostra que participaban en la llamada Conexión Francesa (French Connection, un esquema de narcotráfico a través del cual se pasaban de contrabando toneladas de heroína desde Turquía hasta Estados Unidos, pasando por Francia y Canadá) y la Pizza Connection (una investigación judicial efectuada en Estados Unidos por la DEA y el FBI, en la que se encontró que millones de dólares provenientes de la venta de heroína habían sido blanqueados a través de pizzerías propiedad de miembros de la Cosa Nostra).$^{62}$

El pool finalmente arrestó a 366 presuntos criminales y, en diciembre de 1987, 19 mafiosi fueron condenados a cadena perpetua, además de otras sentencias más breves y 114 absoluciones. ${ }^{63}$ Sin embargo, el éxito del Maxiprocesso no sólo se basó en las penalizaciones sino también en la forma en que se atacó la estructura mafiosa.

En el Manifesto dell' Antimafia, Fernando dalla Chiesa, uno de los líderes y mentes más destacadas de la antimafia (además de hijo del general asesinado Carlo Alberto), argumenta que, en Sicilia, existen cinco fuerzas mafiosas que la antimafia debe desmantelar para tener éxito: legitimidad, invisibilidad material, invisibilidad conceptual, expansividad e impunidad. Siguiendo a Dalla Chiesa, la lucha contra la mafia: a) no es sólo, ni sobre todo, una cuestión de magistrados y fuerzas del orden; b) no es un fenómeno que afecte directamente a sólo tres o cuatro regiones de Italia; c) no puede consistir únicamente en un proceso pacífico e indoloro de educación legal para las generaciones futuras, y d) no se puede agotar en la denuncia. ${ }^{64}$

62 Ron Chepesiuk, The War on Drugs: An International Encyclopedia, Santa Barbara, ABC-Clio, 1999, pp. 105-108.

63 Dickie, op. cit., p. 638.

${ }^{64}$ Nando dalla Chiesa, Manifiesto dell'Antimafia, Torino, Giulio Einaudi editore, 2014, ix. 
Sin embargo, no fue hasta el siglo xxi que se consolidaron estrategias conjuntas con el movimiento antimafia siciliano. En 2017, The Guardian clasificó Palermo como una ciudad resiliente, resultado de una convergencia de fuerzas entre sociedad civil y autoridades que propició un cambio en varias condiciones. ${ }^{65}$ Por ejemplo, la forma en que se profesionalizó la sociedad civil siciliana dedicada a promover la lucha contra la mafia. Como resultado, se estableció una constelación de ONG profesionales y sustentables. Libera y Addiopizzo, ambas organizaciones no gubernamentales, son emblemáticas al respecto.

Según su sitio web, Libera nació “[...] con el propósito de involucrar y apoyar a todos los interesados "en la lucha contra las mafias y el crimen organizado". ${ }^{66}$ Por otro lado, Addiopizzo se ha concentrado en ayudar a las víctimas de extorsión para coordinar, junto con la policía local y los jueces, asesoría para que quienes se hayan visto afectados denuncien los casos de pizzo, la práctica de la extorsión mafiosa.

Otra estrategia es el apoyo electoral a los alcaldes antimafia. Leoluca Orlando es un caso paradigmático. Ha sido alcalde de Palermo de 1985 a 1990, luego de 1993 a 2000, y actualmente desde 2012. También ha sido elegido para la Cámara de Diputados italiana y para el Parlamento Europeo. Desde su primera victoria electoral, Orlando siempre ha mantenido un discurso abiertamente antimafia en sus diferentes cargos. Según Orlando, 1999 fue el primer año sin asesinatos relacionados con la mafia (y con sólo 11 casos sin relación con la mafia en total). Por eso, dijo, en el verano de ese año sintió la "responsabilidad del sobreviviente de contar la historia tal como sucedió". ${ }^{67} \mathrm{El}$ tema da para otro texto.

65 Lorenzo Tondo, "The resurrection of Palermo: how the mafia battlefield became a cultural capital”, The Guardian, 27 de marzo de 2017, https://www.theguardian.com/cities/2017/mar/27/resurrection-palermo-mafia- battlefield-culture-capital

${ }^{66}$ Extraído del sitio de internet de Libera http://www.bottegaliberapalermo.it/chi-siamo/

${ }^{67}$ Leoluca Orlando, Fighting the Mafia and Renewing the Sicilian Culture, Nueva York, Encounter Books, 2001, p. 8. 
La vigente pacificación siciliana se basa, en buena medida, en la construcción de un bloque unificado desde la heterogeneidad que ha servido como plataforma para crear estrategias consensuadas con la autoridad. El resultado ha sido de reducción en la gobernanza criminal.

\section{La Laguna}

El éxito de La Laguna dependió de la convergencia de fuerzas federales, estatales y municipales que atendieron las peticiones de organizaciones sociales, entre las cuales destacaron los grandes empresarios y los colectivos de víctimas apoyados por medios, Iglesia y universidades. Los primeros pedían una policía metropolitana y el combate a los secuestros; los segundos, verdad, justicia y atención especializada.

Para combatir a los Zetas en todo el país, el gobierno federal desplegó nuevas estrategias. La principal fue la creación de los Centros de Fusión de Inteligencia y Operatividad que, en esencia, reunían la información de las diferentes dependencias mexicanas y la proporcionada por los estadounidenses sobre la organización criminal. El objetivo era atacar sus fuentes de ingreso y perseguir a sus jefes.

El viraje federal coincidió con el cambio de gobernador en Coahuila. Rubén Moreira (1. ${ }^{\circ}$ de diciembre de 2011 a 30 de noviembre de 2017) se involucró y armó su estrategia con objetivos específicos: recuperar el penal de Piedras Negras, atender a las víctimas y capturar a los líderes criminales Miguel Ángel y Omar Treviño Morales, el Z40 y el Z42, detenidos en Nuevo León, en julio de 2013 y marzo de 2015, respectivamente. De manera simultánea, el gobierno de Moreira atacó a la organización criminal mediante la reducción de sus ingresos y erosionando sus bases sociales y culturales. Fue un caso exitoso.

En este marco hay que insertar La Laguna. En 2014, las peticiones de los empresarios se hicieron realidad cuando, en una reunión del Gabinete de Seguridad Nacional, se adoptó 
el compromiso de construir un Mando Especial para La Laguna encabezado por la Secretaría de la Defensa Nacional. Con ello se resolvió la fragmentación del mando. Se creó la Fuerza Metropolitana, un cuerpo de seguridad con mando de un militar y con policías de Coahuila, Durango y la federación. El Mando Especial ha funcionado porque resolvió los problemas asociados con el conflicto creado por la competencia de municipios de dos estados. También se creó la Unidad Metropolitana Antisecuestros. El resultado ha sido una caída notable en el número de homicidios dolosos y desaparecidos. Reiteramos que una razón del éxito es la permanencia del Consejo Cívico de las Instituciones Laguna que monitorea permanentemente la incidencia delictiva.

Los colectivos de víctimas también lograron avances emulados en otras partes de México. Han sido pioneros en las campañas de difusión de información sobre desaparecidos, gestiones ante las autoridades, búsquedas en campos y zonas de exterminio, foros de análisis, propuesta de instrumentos legales, etcétera. Un ejemplo concreto es la creación, en septiembre de 2012, del Grupo Autónomo de Trabajo (GAT), un espacio de encuentro entre colectivos de víctimas y gobierno estatal que facilitó la colaboración sobre diferentes aspectos. En suma, Coahuila se ha convertido en ejemplo nacional para el diálogo entre víctimas y autoridades, y para la creación de legislación y política pública.

Los Zetas fueron fragmentados y descabezados y se redujo la violencia en el estado de Coahuila. Sin embargo, en La Laguna sigue presente el cartel de Sinaloa, que se maneja con mucha mayor discreción. En todo caso, a mediados de 2021 se mantiene controlada la gobernanza criminal.

Conclusiones. Comparación de Chicago, Sicilia y La LAGUNA

En los tres casos abordados en este artículo, resalta una comunión de visiones y voluntades de la sociedad civil y las au- 
toridades para disputar la legitimidad política local al grupo criminal. Además, comparten algunas similitudes en la forma de reducir la violencia y la gobernanza criminal.

En primer lugar, en las tres instancias hubo un grupo criminal dominante dedicado a actividades ilícitas que contaba con el respaldo de una parte de la sociedad organizada para crear una autoridad criminal de facto. En otras palabras, había gobernanza criminal a nivel local.

La segunda similitud es que la hegemonía del grupo criminal local supuso la existencia de un sistema que determinaba el tipo y la forma de las relaciones sociales locales. Dicho esto, también influyeron factores nacionales y globales que sólo mencionamos muy brevemente.

Finalmente, hay presencia de un movimiento social que, aliado con la autoridad legal, contrarrestó la gobernanza criminal. Es decir, en los tres casos ocurrió una construcción, densificación y consolidación de capital social positivo, inspirado y diseñado para disminuir la gobernanza criminal dominante y el capital social negativo que produjo en cada caso. ¿Cómo describir ese capital social positivo?

A pesar de la heterogeneidad en latitudes, contextos y temporalidades, la constelación de actores sociales que forman dicho movimiento es similar. En los tres casos participan periodistas, académicos, empresarios, organizaciones religiosas, organizaciones de la sociedad civil y víctimas individuales o agrupados en colectivos. Cada sector lo hizo apoyándose en sus propios relatos, intereses y liderazgos, aunque compartiendo el deseo de reducir la gobernanza criminal y la disposición de acercarse a funcionarios públicos comprometidos con el combate del grupo criminal local.

Esta confluencia de actores permitió la creación de islotes de paz: polígonos geográficos donde hay relaciones sociales que privilegian el respeto a los derechos humanos y al Estado de derecho. En otras palabras, capital social positivo. Estos espacios generan una legitimidad propia que se expande y crea geografías de paz donde se construyen resistencias más amplias a la gobernanza criminal, que incluye la com- 
plejísima relación con aquellas personas y grupos de la comunidad que simpatizan con o respaldan a los criminales.

El éxito en la reducción de la violencia criminal es indudable en los tres casos. En Chicago cumplieron el limitado objetivo de encarcelar a Capone y empezó la construcción de fórmulas cada vez más elaboradas para enfrentar la criminalidad. En Italia, el número de homicidios de asociaciones de tipo mafioso disminuyó casi $80 \%$ entre 1992 y 2012 , y fue en Sicilia donde ocurrió la mayor reducción. ${ }^{68}$ Según Massari, los homicidios cometidos por la Cosa Nostra pasaron de 152 en 1992 a sólo 10 en $2010 .{ }^{69}$ La disminución porcentual en este caso equivale a $93.4 \%$. Ningún otro grupo criminal mafioso redujo tanto la dimensión de la violencia en ese periodo. Finalmente, en La Laguna, a partir de 2012 cayó el número de homicidios y desapariciones. En 2011 hubo 1060 homicidios dolosos y 139 en 2018.

Aun y cuando la violencia asociada a dinámicas de la gobernanza criminal local se redujo drásticamente en los tres casos, aún hay retos en la recuperación de la memoria, reparación del daño y acceso a la justicia. Además, no se eliminó de manera permanente a los grupos criminales, aunque sí se fortaleció el convencimiento de que los mercados ilegales no son necesaria e irrenunciablemente violentos.

$* * * * * * * * * * *$

El exitoso modelo, ¿podrá reproducirse en otros centros urbanos? En Chicago, Sicilia y La Laguna la convergencia y el cabildeo tuvieron una lógica espontánea. ¿Podría inducirse ese proceso "desde fuera"?

El 11 de marzo de 2019, la Secretaría de Educación, Ciencia, Tecnología e Innovación de la Ciudad de México (Sectei)

68 United Nations Office on Drug and Crime, UnOdc, Global Study on Homicide 2013. Trends, Contexts, Data, Vienna, United Nations, 2014, p. 44.

69 Monica Massari, "Guns in the Family-Mafia violence in Italy" (cap. 4), en Small Arms Survey. Everyday Dangers, Geneva, Cambridge, University Press, 2013, p. 83. 
dio inicio al proyecto Red Ecos, que consiste en convocar a instituciones de educación superior públicas y privadas, dependencias gubernamentales, centros públicos de investigación, organismos de la sociedad civil y distintos actores del sector productivo para integrar coaliciones interdisciplinarias e interinstitucionales que, después de estudiar un problema, propongan al gobierno capitalino políticas públicas.

Poco después, la Sectei invitó a El Colegio de México a coordinar el esfuerzo por estudiar la violencia en la capital para proponer soluciones. La presidenta de la institución, Silvia Giorguli, propuso al Seminario sobre Violencia y Paz de El Colegio de México. Así fue como los autores de este texto empezamos a explorar la posibilidad de que el modelo descrito anteriormente pueda ser reproducido en la Ciudad de México. En mayo de 2021, 13 instituciones presentamos una propuesta al Consejo Nacional de Ciencia y Tecnología (Conacyt) para tres años. ${ }^{70}$

Entre los primeros objetivos de la propuesta está la construcción de confianza y métodos de trabajo entre las 13 instituciones participantes, la comprensión del tejido social a nivel macro (alcaldías y ciudad) y micro (el estudio de la capacidad que tienen los Puntos de Innovación Libertad, Arte, Educación y Saberes -Pilares- de la Ciudad de México para convertirse en islotes de paz) y la preparación de una pro-

${ }^{70}$ Las instituciones son, en orden alfabético, el Centro de Investigación en Ciencias de Información Geoespacial (Centro Geo); el Centro de Investigación y de Estudios Avanzados del Instituto Politécnico Nacional (Cinvestav); el Centro de Investigaciones y Estudios Superiores en Antropología Social (CIEsas); el Colectivo para el Análisis de la Seguridad con Democracia A.c. (Casede); la Fundación para el Estudio de la Seguridad y Gobernanza A.c. (Fesego); el Programa de Licenciatura en Ciencia Forense de la Universidad Nacional Autónoma de México (UnAm); el Programa Pilares de la Ciudad de México; la Secretaría de Educación, Ciencia, Tecnología e Innovación de la Ciudad de México (Sectei), coordinadora de la Redecos; la Secretaría de Seguridad Ciudadana de la Ciudad de México; Seguridad y Paz Ciudadana, A.C (Seypaz); el Seminario sobre Violencia y Paz de El Colegio de México (instancia coordinadora), y la Universidad Iberoamericana (UIA). 
puesta que nos permita tener un acercamiento con los líderes de los seis actores sociales mencionados en el texto para la elaboración de una agenda de seguridad mínima. En el trasfondo está la formulación de un marco teórico flexible y riguroso que nos permita ir resolviendo los múltiples problemas teóricos y prácticos asociados con una urbe gigantesca.

En textos posteriores analizaremos la manera en que fuimos resolviendo estos retos que abordamos con una tesis central: para conquistar la paz debemos comprender la lógica de la violencia criminal.

\section{REFERENCIAS}

Aguayo, Sergio y Jacobo Dayán, El yugo Zeta: norte de Coahuila 2010-2011, México, El Colegio de México, 2018.

Aguayo, Sergio y Jacobo Dayán, "Reconquistando" La Laguna, México, El Colegio de México, 2020.

Arjona, Ana, Nelson Kasfir y Zachariah Mampilly, "Introduction”, en Ana Arjona, Nelson Kasfir y Zachariah Mampilly, Rebel Governance in Civil War, Nueva York, Cambridge University Press, 2015.

Arriagada, Irma, "Capital social: potencialidades y limitaciones analíticas de un concepto", Estudios Sociológicos, xxI, núm. 3 (2003), pp. 557-584.

Azaola, Elena, "La violencia de hoy, las violencias de siempre", Desacatos, 40, septiembre-diciembre (2012), pp. 159-170.

Bergreen, Laurence, Capone: The Man and the Era, Nueva York, Simon and Schuster (Kindle edition), 1996, 724 pp.

BLok, Anton, The Mafia of a Sicilian village, 1860-1960: A study of violent peasant entrepreneurs, Londres, Harper and Row, 1974.

Bourdieu, Pierre, "The Forms of Capital", en John Richardson (ed.), Handbook of Theory and Research for the Sociology of Education, Nueva York, Greenwood Press, 1986.

Burns, Ken y Lynn Novick (dirs.), Prohibition, "Una nación de hipócritas” (cap. 3) (serie documental), Netflix, 2011. 
Callahan, William, "Social Capital and Corruption: Vote Buying and the Politics of Reform in Thailand", Perspectives on Politics, 3 (3), (2005), pp. 495-508.

Chepsiuk, Ron, The War on Drugs: An International Encyclopedia, Santa Barbara, ABC-Clio, 1999.

Correa-Cabrera, Guadalupe, Los Zetas Inc. La corporación delictiva que funciona como empresa transnacional, México, Planeta, 2018.

Corvaia, Marco, Pino se lo aspettava. Il racconto de la vida e della morte di Padre Puglisi, Palermo, Navarra Editore, 2012.

Dalla Chiesa, Nando, Manifiesto dell'Antimafia, Torino, Giulio Einaudi editore, 2014.

Daniel, Jan, Criminal Governance and Insurgency, CejISs, 4, 2015.

Desmond Arias, Enrique, Criminal Enterprises and Governance in Latin America and the Caribbean, Cambridge, University Press, 2017, 302 pp.

Dickie, John, Historia de la mafia. Cosa Nostra, 'Ndrangheta, Camorra de 1860 al presente, Barcelona, Penguin Random House, 2015.

Garza Ramos, Javier, "Under Fire in Mexico", Harvard Review of Latin America, Journalism of the Americas (primavera|verano de 2011), https://revista.drclas.harvard.edu/book/underfire-mexico

Garzón, Adela, "Familismo y creencias políticas", Psicología Política, núm. 17, noviembre de 1998, pp. 101-128.

Garzón, Juan Carlos, "El futuro del narcotráfico y los traficantes en América Latina”, en Sergio Aguayo y Raúl Benítez (eds.), Atlas de la Seguridad y la Defensa en México 2012, México, Casede/Open Society, 2012.

Gosch, Martin A. y Richard Hammer, The Last Testament of Lucky Luciano: The Mafia Story in His Own Words, Enigma Books (edición Kindle), 1975, posiciones, 2033-2034.

Granovetter, Mark, "The Strength of Weak Ties", American Journal of Sociology, 78, 6, Chicago, The University of Chicago Press, 1973, pp. 1360-1380.

Grillo, Ioan, Gangster Warlords. Drug Dollars, Killing Fields and the New Politics of Latin America, Nueva York, Bloomsbury, 2017.

Landesco, John, Organized Crime in Chicago. Part III of the Illinois Crime Survey, 1929, Chicago, The University of Chicago Press, 1968. 
Lessing, Benjamin, "Conceptualizing Criminal Governance”, Perspectives on Politics, 2020, 1-20. https://doi.org/10.1017/S15375 92720001243

Libera, "About Us", en Libera International (sitio de internet) (s/f), http:/ / www.liberainternational.eu/?page_id=10

McDonough, Daniel, "Chicago Press Treatment of the Gangster, 1924-1931”, Illinois Historical Journal, vol. 82, núm. 1, (primavera de 1989), pp. 17-32, https:/ / www.jstor.org/stable/40192250

Massari, Monica, "Guns in the Family-Mafia violence in Italy" (cap. 4), en Small Arms Survey. Everyday Dangers, Geneva, Cambridge, University Press, 2013.

Matos, Heloiza, "O lado escuro do capital social: implicações sociais e políticas”, Libero, 12 (23), 2009, pp. 53-62.

Orlando, Leoluca, Fighting the Mafia and Renewing the Sicilian Culture, Nueva York, Encounter Books, 2001.

Paoli, Letizia, Mafia Brotherhoods: Organized Crime, Italian Style, Oxford, University Press, 2003.

Perry, Douglas, Eliot Ness: The Rise and Fall of an American Hero, EE.UU., Viking (edición Kindle), 2014, 337 pp.

Portes, Alejandro, "Capital social: origens e aplicações na sociologia contemporânea”, Revista sociologia, problemas e práticas, (33), Lisboa (2000), pp. 133-158.

Putnam, Robert, Making Democracy Work: Civic Traditions in Modern Italy, Princeton, University Press, 1993.

ReAvill, Gill, Mafia Summit:J. Edgar Hoover, the Kennedy Brothers and the Meeting that Unmasked the Mob, Nueva York, Thomas Dunne Books (edición Kindle), 2015.

Rubio, Mauricio, Homicidios, justicia, mafias y capital social: otro ensayo sobre la violencia colombiana, Bogotá, Centro de Estudios sobre Desarrollo Económico, Universidad de los Andes, 1996, http:/ / hdl.handle.net/1992/40933

Sánchez, Víctor, Manuel Pérez y Jorge Verástegui, Formación y desarrollo de los colectivos de búsqueda de personas desaparecidas en Coahuila: Lecciones para el futuro, México, El Colegio de México, 2012.

Santino, Umberto, Breve Storia della Mafia e dell 'Antimafia, Palermo, Di Girolamo, 2011. 
Schneider, Jane y Peter Schneider, Reversible Destiny. Mafia, Antimafia, and the Struggle for Palermo, California, University of California Press, 2003.

Schneider, Jane y Peter Schneider, "Mafia, Antimafia, and the Plural Cultures of Sicily", Current Anthropology, vol. 4 (agostooctubre de 2005), pp. 501-520.

Schneider, Jane y Peter Schneider, "Civil Society Versus Organized Crime”, Critique of Anthropology, 21, 4, 2001, pp. 427-446.

Schlesinger, Arthur M., Robert Kennedy and his Times, Boston y Nueva York, Houghton Mifflin, 2018, 1092 pp.

"Taking Down the Mob" (cap. 4), Inside the American Mob (serie documental), National Geographic, 2013.

Tondo, Lorenzo, "The resurrection of Palermo: how the mafia battlefield became a cultural capital", The Guardian, 27 de marzo de 2017, https://www.theguardian.com/cities/2017/mar/27/ resurrection-palermo-mafia-battlefield-culture-capital

Trejo, Guillermo y Sandra Ley, Votes, Drugs, and Violence: The Political Logic of Criminal Wars in Mexico, Cambridge, University Press, 2020.

United Nations Office on Drug and Crime, UnODC, Global Study on Homicide 2013. Trends, Contexts, Data, Vienna, United Nations, 2014, 164 pp., https://www.unodc.org/documents/gsh/pdfs/ 2014_GLOBAL_HOMICIDE_BOOK_web.pdf

VARESE, Federico, Vita di mafia. Amore, morte e denaro nel cuore del crimine organizzato, Torino, Giulio Einaudi editore, 2017.

Von Lampe, Klaus (comp.), Definitions of Organized Crime (sitio de internet), www.organized-crime.de/organizedcrimedefinitions.htm 